International Journal of Quantum Chemistry 114:1041-1047 (2014)

DOI: $10.1002 / q u a .24623$ 


\title{
Effective atomic orbitals - a tool for understanding electronic structure of molecules
}

\author{
I. Mayer* \\ Research Centre for Natural Sciences, \\ Hungarian Academy of Sciences, H-1525 Budapest, P.O.Box 17, Hungary
}

\begin{abstract}
It is discussed that one can obtain effective atomic orbitals in quite different theoretical frameworks of Hilbert-space and 3D analyses. In all cases one can clearly distinguish between the orbitals of an effective minimal basis set and orbitals which are only insignificantly occupied. This observation makes a solid theoretical basis beyond our qualitative picture of molecular electronic structure, described in terms of minimal basis atomic orbitals having decisive participation in bonding, and may be considered as a quantum chemical manifestation of the octet rule. For strongly positive atoms like the hypervalent sulfur, some weakly occupied orbitals reflecting "back donation" can also be identified. From the conceptual point of view, it is very important that atomic orbitals of characteristic shape can be obtained even by processing the results of plane wave calculations in which no atomcentered basis orbitals were applied.

The different types of analyses (Hilbert-space and 3D) can be done on equal footing, performing quite analogous procedures, and they exhibit an unexpected interrelation, too.
\end{abstract}

*mayer@chemres.hu, mayer.istvan@ttk.mta.hu 


\section{Introduction}

Atomic orbitals (AOs) represent an important concept in the theory of atomic and molecular physics. Historically, they first appeared as exact solutions of the Schrödinger equation of the free hydrogen atom, then they have been obtained as solutions in the Hartree-Fock (HF) approximation for the free many-electron atoms. The HF "canonical" orbitals are qualitatively similar to the hydrogenic ones, but without the "accidental" degeneracy of the $s, p$ etc. orbitals of the same principal quantum number. Accounting for some peculiarities in the order of the orbital energies and the corresponding shellfilling scheme (like $3 d v s$. $4 s$ for transition metals), the use of the AO concept permits one to rationalize the periodic system of the elements and to specify different electronic states of the free atoms.

Started with the classical work of Heitler and London, the first numerical calculations on molecular systems (basically $\mathrm{H}_{2}$ in the very first period) were performed by using the orbitals of free atoms (maybe squeezed and/or polarized) as the indispensable tools. Then the "founding Fathers" of quantum chemistry (who had not the possibility to perform large scale calculations but had the ability of deep thinking) introduced a number of qualitative/semiquantitative concepts, like "linear combination of atomic orbitals" (LCAO), hybridization, delocalization etc., until now forming the basis for our qualitative interpretation of electronic structure of molecules - always in terms of different atomic orbitals. The first $a b$ initio calculations (and, in fact, also the semiempirical ones) have been performed in terms of the "minimal basis" of atomic orbitals, either those of the free atoms, or by using some approximation to them in terms of Slater-type or (contracted) Gaussian-type "basis orbitals".

With the development of the computers and computational techniques, these concepts basically disappeared from the quantum chemical discourse, much more oriented to the numbers - energies, geometrical parameters, etc. LCAO has "survived" in the sense that most calculations use atom-centered basis sets, although in the last years more and more calculations are performed by applying plane wave basis sets, i.e., without using explicitly the LCAO concept. ${ }^{1}$ By the use of larger and larger basis sets, the concept of well defined atomic orbitals making up the molecular ones does not appear in a routine quantum chemical calculation - although it is probably crucial for a proper understanding of the results of these calculations. For instance, to interpret planarity of the benzene molecule, one can hardly avoid the concept of $s p^{2}$ hybridization of the carbon's atomic orbitals.

\footnotetext{
${ }^{1}$ In the plane wave calculations one often uses pseudo-potentials to replace inner shell electrons. That is, of course, in some sense also an application the LCAO concept.
} 
In such a situation it represents a conceptual interest how the classical picture of minimal basis core and valence orbitals can be recovered by an appropriate a posteriori analysis of wave functions - the present paper is devoted to this problem. For sake of simplicity, we shall concentrate on closed shell systems treated at the single determinant (Hartree-Fock or DFT) level of theory.

As known, there are two main approaches for doing a posteriori analysis of wave functions: the Hilbert-space analysis and the 3D analysis [1]. In the former case one considers the atom in molecule as an entity defined by the nucleus and the basis orbitals assigned to it, while in the latter one the atom is defined as the nucleus and a disjunct or "fuzzy" domain of the threedimensional physical space around it. In our analysis we shall pursue both types of analysis simultaneously, by using a common formalism, and then we shall discuss some interrelations between these two approaches.

Of course, thee were several approaches to the problem of atomic orbitals within a molecule during the decades, using different theoretical approaches and introducing different criteria to determine the AOs. We should first of all mention the "effective hybrids" of McWeeny [2], diagonalizing the intraatomic block of the density matrix (in an orthonormalized basis), our approach may be considered a direct generalization of which. The "modified atomic orbitals" of Heinzmann and Ahlrichs [3] were based on the concept of Roby's charge [4], and minimized the "unassigned charge", not accounted for by the minimal basis modified AOs. In the "natural atomic orbital" (NAO) analysis of Weinhold an coworkers [5,6], a rather complex recipe is used in order to obtain a set of NAOs which can be assigned core, valence or "Rydberg" character and are also used to perform the natural population analysis". While one often can get useful chemical insight by using these NAO-s, we feel lacking a clear mathematical criterion (some target functional) behind them. Also, as the final NAO-s of a whole molecule are obtained orthonormalized, they do not carry any direct information about the (chemically very important, in our opinion) effects of interatomic overlap. In [7] we have proposed to use the Magnasco-Perico localization criterion [8] (maximization of Mulliken's net atomic population) in order to determine the molecular orbitals from which effective AOs can be obtained; then the approach was generalized to an arbitrary Hermitian bilinear localization functional [9]; actually the use of Mulliken's gross populations was also tested numerically (and rejected). The approach has been generalized to the 3D analysis, too [10-13]. The "free" (or "broken") valences obtained in the framework of the "domain averaged Fermi hole" (DAFH) formalism of Ponec and coworkers [14-15] are entities very close to the atomic orbitals forming chemical bonds; despite the fact that the DAFH formalism is an 
explicitly two-electron scheme and uses the second order density matrix, in the single determinant (Hartree-Fock or Kohn-Sham) case it reduces to the calculation of group functions exactly in the manner of our theory $[16,17]$, showing that these approaches are closer to each other than it would appear at the first sight.

\section{Atomic orbitals from molecular wave functions}

The solutions of the HF or KS equations are delocalized canonical molecular orbitals (MOs). However exactly the same wave function can be described also by different sets of localized molecular orbitals. We shall use a special localization scheme permitting to restore the AO-s actually participating in building the MO-s.

Atomic parts of the molecular orbitals

At first we define the MO-s as representing sums of some "atomic parts"

$$
\varphi=\sum_{A} \varphi^{A}
$$

The definition of the atomic part $\varphi^{A}$ of the $\mathrm{MO} \varphi$ depends on whether one uses Hilbert-space or 3D analysis.

In the Hilbert-space analysis one starts from the LCAO expansion of the $\mathrm{MO}$

$$
\varphi=\sum_{\mu} c_{\mu} \chi_{\mu}
$$

and only the basis orbitals centered on atom $A$ are conserved in the expansion to form the atomic part $\varphi^{A}$ of the $\operatorname{MO} \varphi$ :

$$
\varphi^{A}=\sum_{\mu \in A} c_{\mu} \chi_{\mu}
$$

In the case of the Bader's "atoms in molecules" (AIM) analysis - or, in general, if the 3D physical space is decomposed into the disjoint atomic domains $\Omega_{A}$ - the definition of the atomic part of an orbital $\varphi$ is simply

$$
\varphi^{A}= \begin{cases}\varphi(\vec{r}) & \text { if } \vec{r} \in \Omega_{A}, \\ 0 & \text { otherwise . }\end{cases}
$$

In the "fuzzy atoms" analysis there are no sharp boundaries of the atoms. In this case we define in every point $\vec{r}$ of the space the atomic weight functions $w_{A}(\vec{r})$ that are large inside the respective atom $A$, small outside it, and satisfy

$$
\sum_{A} w_{A}(\vec{r})=1, \quad \forall \vec{r} .
$$


Then the atomic part of the orbital $\varphi$ is defined as

$$
\varphi^{A}=w_{A}(\vec{r}) \varphi(\vec{r})
$$

The localization procedure and the effective atomic orbitals

We perform a separate localization for each atom, by requiring

$$
\delta M_{i}^{A}=\delta \frac{\left\langle\varphi_{i}^{A} \mid \varphi_{i}^{A}\right\rangle}{\left\langle\varphi_{i} \mid \varphi_{i}\right\rangle}=0
$$

i.e., the atomic part $\varphi_{i}^{A}$ of each localized orbital $\varphi_{i}$ must have a maximal (at least stationary) norm. ${ }^{2}$

By writing the localized orbital as a linear combination of the canonical ones:

$$
\varphi_{i}=\sum_{j=1}^{n} U_{j i}^{A} \psi_{j}
$$

where $n$ is the number of the (doubly filled) occupied orbitals, the stationarity requirement, in a standard manner, leads to the eigenvalue equation

$$
\mathbf{Q}^{A} \mathbf{U}^{A}=\mathbf{U}^{A} \mathbf{M}^{A}
$$

Here the Hermitian matrix $\mathbf{Q}^{A}$ has the elements

$$
Q_{i j}^{A}=\left\langle\psi_{i}^{A} \mid \psi_{j}^{A}\right\rangle
$$

where $\psi_{i}^{A}$ is the atomic part of the (normalized) canonical orbital $\psi_{i}$, defined in the manner discussed above, and $\mathbf{M}^{A}$ is the diagonal matrix of the eigenvalues

$$
\mathbf{M}^{A}=\operatorname{diag}\left\{M_{i}^{A}\right\} .
$$

When doing this derivation, we have taken into account that forming the atomic part of an orbital is a linear procedure in each case discussed above, so expansion (8) holds for the atomic parts of the respective orbitals, too, and one can write

$$
\varphi_{i}^{A}=\sum_{j=1}^{n} U_{j i}^{A} \psi_{j}^{A}
$$

These equations were obtained for the different schemes of analysis [7-11] independently from each other, although a general mathematical formalism

\footnotetext{
${ }^{2}$ Of course, one might distinguish the sets localized orbitals $\left\{\varphi_{i}\right\}$ corresponding to different atoms by introducing an additional subscript/superscript, but that would lead to very cumbersome notations.
} 
has also been discussed [9]. As can be seen here, one should solve quite similar equations independently of the type of analysis. Also, it is common that the atomic parts of the localized orbitals after renormalization define an orthonormalized set of effective atomic orbitals $\chi_{i}^{A}$ with the eigenvalues $M_{i}$ being their occupation numbers (net atomic populations of the respective localized orbitals). This means that

$$
\chi_{i}^{A}=\frac{1}{\sqrt{M_{i}^{A}}} \varphi_{i}^{A} .
$$

Orbitals $\chi_{i}^{A}$ are indeed orthonormalized:

$$
\begin{aligned}
\left\langle\chi_{i}^{A} \mid \chi_{j}^{A}\right\rangle & =\frac{1}{\sqrt{M_{i}^{A} M_{j}^{A}}}\left\langle\varphi_{i}^{A} \mid \varphi_{j}^{A}\right\rangle=\frac{1}{\sqrt{M_{i}^{A} M_{j}^{A}}} \sum_{k, l=1}^{n} U_{k i}^{A *}\left\langle\psi_{k}^{A} \mid \psi_{l}^{A}\right\rangle U_{l j} \\
& =\frac{1}{\sqrt{M_{i}^{A} M_{j}^{A}}} \sum_{k, l=1}^{n}\left(\mathbf{U}^{A \dagger}\right)_{i k} Q_{k l}^{A} U_{l j}=\frac{1}{\sqrt{M_{i}^{A} M_{j}^{A}}} M_{i}^{A} \delta_{i j}=\delta_{i j},
\end{aligned}
$$

owing to the fact that the unitary matrix $\mathbf{U}^{A}$ diagonalizes matrix $\mathbf{Q}^{A}$.

One can see by inspection that orbitals $\chi_{i}^{A}$ represent the results that one obtains by performing Löwdin's canonic orthogonalization ${ }^{3}[18]$ of the atomic components $\psi_{i}^{A}$ of the canonical orbitals $\psi_{i}$.

In the case of a Hilbert-space analysis, the number of the effective atomic orbitals of the given atom, having nonzero occupation numbers, is limited by the smaller of the number of the basis orbitals of that atom and the number of molecular orbitals in the molecule. In the 3D formalism one gets, in general, as many effective AO-s for each atom, as is the number of MO-s in the molecule.

\section{The effective AO-s as natural hybrids}

It is known that the first order spinless density matrix can be expressed by the natural orbitals $\psi_{i}(\vec{r})$ and their occupation numbers $n_{i}$ as

$$
\varrho_{1}\left(\vec{r}, \vec{r}^{\prime}\right)=\sum_{i} n_{i} \psi_{i}(\vec{r}) \psi_{i}^{*}\left(\vec{r}^{\prime}\right)
$$

One can define the (intra)atomic part of the spinless first-order density matrix in terms of the atomic parts $\psi_{i}^{A}$ of the natural orbitals, formed according to the schemes discussed above:

$$
\varrho_{1}^{A}\left(\vec{r}, \vec{r}^{\prime}\right)=\sum_{i} n_{i} \psi_{i}^{A}(\vec{r}) \psi_{i}^{A *}\left(\vec{r}^{\prime}\right)
$$

\footnotetext{
${ }^{3}$ Not to be confused with Löwdin's more common "symmetric" orthogonalization.
} 
In the case of a single closed shell determinant wave function, all the occupation numbers $n_{i}$ are equal either 2 or 0, and Eq. (16) becomes

$$
\varrho_{1}^{A}\left(\vec{r}, \vec{r}^{\prime}\right)=2 \sum_{i}^{n} \psi_{i}^{A}(\vec{r}) \psi_{i}^{A *}\left(\vec{r}^{\prime}\right)
$$

In the closed-shell single determinant case one has the unitary invariance property; it is easy to check that it also holds to Eq. (17). That means that any set of orthonormalized occupied orbitals may equally well be used in this expressions, including those in Eq. (12), obtained by solving the eigenvalue equation of matrix $\mathbf{Q}^{A}$ for the given atom:

$$
\varrho_{1}^{A}\left(\vec{r}, \vec{r}^{\prime}\right)=2 \sum_{i}^{n} \varphi_{i}^{A}(\vec{r}) \varphi_{i}^{A *}\left(\vec{r}^{\prime}\right)
$$

It is known that the first order spinless density matrix $\varrho_{1}\left(\vec{r}, \vec{r}^{\prime}\right)$ is (up to a factor of $\frac{1}{2}$ ) the kernel of the projection operator $\hat{\rho}$ onto the subspace of the occupied orbitals and the eigenvectors of the latter are the natural orbitals; using the "bra-ket" notations that can be written as

$$
\hat{\rho}\left|\psi_{j}\right\rangle=\sum_{i} n_{i}\left|\psi_{i}\right\rangle\left\langle\psi_{i} \mid \psi_{j}\right\rangle=\sum_{i} n_{i} \delta_{i j}\left|\psi_{i}\right\rangle=n_{j}\left|\psi_{j}\right\rangle
$$

Equation (13) can be written as $\varphi_{i}^{A}=\sqrt{M_{i}} \chi_{i}^{A}$, by using which Eq. (18) can be rewritten as

$$
\varrho_{1}^{A}\left(\vec{r}, \vec{r}^{\prime}\right)=2 \sum_{i}^{n} M_{i} \chi_{i}^{A}(\vec{r}) \chi_{i}^{A *}\left(\vec{r}^{\prime}\right)
$$

This can also be considered a kernel of an integral operator, and one can write, in analogy to Eq. (19)

$$
\hat{\rho}^{A}\left|\chi_{j}\right\rangle=2 \sum_{i}^{n} M_{i}\left|\chi_{i}^{A}\right\rangle\left\langle\chi_{i}^{A} \mid \chi_{j}^{A}\right\rangle=2 \sum_{i}^{n} M_{i} \delta_{i j}\left|\chi_{i}^{A}\right\rangle=2 M_{j}\left|\chi_{j}^{A}\right\rangle
$$

where the orthonormalization property Eq. (14) has been utilized. This result indicates that the effective atomic orbitals $\chi_{i}^{A}$ may be considered direct generalizations of the "natural hybrid orbitals" introduced by McWeeny [2], with the occupation numbers $2 M_{i}$ representing their net atomic populations.

In the case of a correlated wave function, the present approach can easily be generalized by considering the atomic part of the spinless first order density matrix, defined in Eq. (16), as a kernel of an integral operator and solving the eigenvalue equation

$$
\hat{\rho}^{A}\left|\chi_{j}^{A}\right\rangle=\sum_{i} n_{i}\left|\psi_{i}^{A}\right\rangle\left\langle\psi_{i}^{A} \mid \chi_{j}^{A}\right\rangle=2 M_{j}\left|\chi_{j}^{A}\right\rangle,
$$

giving the effective atomic orbitals in the correlated case. 


\section{The effective minimal basis}

Experience shows that for non-hypervalent systems there are always as many effective $A O-s$ with $M_{i}$ values significantly greater than zero, as is the number of AO-s in the classical minimal basis of the atom. They define an effective minimal basis of the atom. This is the case at any types of treatment - Hilbert-space[7,9], fuzzy-atoms [11] as well as for Bader's AIM [13] — for all "normal valence" compounds, while for hypervalent compounds a small "shoulder" is observed on the curves of occupation numbers, reflecting the effects of "back donation" to (usually $d$-type) orbitals. It is important to stress that this observation is not connected with the use of any atomcentered basis set, but is valid even for the pure plane wave calculations [12], so it indeed reflects some general property of molecular wave functions.

This observation may be considered as a quantum chemical manifestation of the octet rule: for normal valence non-hydrogenic atoms one has (besides the core shells) four orbitals in a classical minimal basis. The observation mentioned means that the number of molecular orbitals with a considerable contribution from the valence type basis orbitals of the given atom is also four; in a closed-shell molecule these four orbitals carry eight electrons in accord with the octet rule. (The back-donation effects for hypervalent atoms do somewhat modulate but not invalidate this conclusion.)

\section{Some examples}

During the years we have considered the effective AO-s in different frameworks. First we have obtained effective AO-s in classical Hilbert-space analysis $[7,9]$, for which a free program has also made available [19], then for the "fuzzy atoms" analysis [11] of standard wave functions built up from atomcentered basis sets. Most recent are the effective AO-s obtained in "fuzzy atoms" analysis accomplished for calculations using plane wave basis [12], as well as the calculations in the framework of the Bader's AIM analysis [13].

Here we are going to consider only a few examples. Figures 1 and 2 show typical results obtained in the framework of the Hilbert-space analysis, accomplished with the program [19] mentioned. These figures display the occupation numbers ${ }^{4}$ calculated for the different atoms of the glycine and $\mathrm{CH}_{3} \mathrm{SO}_{2}$ molecules, respectively, both by using the cc-pVTZ basis set. (According to our experience, this basis is very well suited for a posteriori analyses of the quantum chemical results, as it is well-balanced and combines flexibility with a pronounced atomic character of the basis functions.)

\footnotetext{
${ }^{4}$ On these graphs the quantity $M_{i}^{A}$ is twice of that discussed above.
} 
It can be seen that all the hydrogen atoms exhibit only a single orbital with non-negligible occupation number, in accord with the fact that hydrogens have only one valence orbital. The carbon, nitrogen and oxygen atoms have five effective AO-s with significant occupation numbers - again in full agreement with the classical chemical notions: one $1 s$ core and four $2 s-2 p$ valence orbitals. Similarly to that, the "normal valence" chlorine atom in the $\mathrm{CH}_{3} \mathrm{SO}_{2}$ molecule has nine effective $\mathrm{AO}$-s: there is an additional completely filled $2 s-2 p$ shell as compared with $\mathrm{C}, \mathrm{N}$ or $\mathrm{O}$, so the valence orbitals belong to the $3 s-3 p$ shell.

However, the hypervalent sulfur, that has a formal valence equal six, exhibits four additional effective AO-s with small but by far not negligible occupation numbers; they practically consist of $d$-type atomic basis orbitals only. Considering hypervalency, it is worth mentioning that the occupation numbers displayed on the figure represent the net orbital occupations of the effective AO-s; although these are small, the contribution of these orbitals to the total valence is quite considerable and the resulting valence of 5.816 is approaching the formal value of six-see Table $1 .^{5}$

Figure 3 shows the four strongly occupied valence orbitals and the first four weakly occupied ( $d$-type) orbitals of the sulfur atom in the $\mathrm{SF}_{6}$ molecule, extracted by the "fuzzy atoms" analysis of the plane wave DFT calculation [12]. The orbitals that were obtained without using any atom centered basis set resembles completely the classical $s p$ valence basis, if one takes into account that their outer parts are "cut" in accordance with the quickly decreasing atomic weight function $w_{A}(\vec{r})$. The emergence of the atomic $d$-orbitals from the results of plane wave calculations is also quite reassuring.

Figure 4 shows the effective AO-s of the carbon atom in methane molecule and their occupation numbers, obtained from a Bader-analysis of a B3LYP/cc-pVTZ calculation. Again, the orbitals essentially look as usual, only they are strictly limited to the AIM atomic domain with sharp boundaries - as they should.

From our point of view, we may call most appropriate those basis sets and 3D atomic definitions which show the sharpest drop of the occupation numbers $M_{i}$ after the functions of the minimal basis orbitals for conventional organic compounds. For such basis sets (atom definitions) the appearance

\footnotetext{
${ }^{5}$ The partial valences displayed in this table have been calculated as sums of the bond orders $[20,21]$ formed by the atomic basis orbitals of the given type. The results are in agreement with the chemical expectations: hydrogen forms one bond by its $1 s$-type orbital, for carbon contribution of each of the $2 s$ and $2 p$ orbitals is nearly one; the bonds of the oxygen and fluorine are formed mainly by their $p$-orbitals. The (rather positive) hypervalent sulfur forms four bonds like carbon, and the additional two are due to the weakly populated but still present- $d$-orbitals resulted from "back-donation".
} 
of shoulders in the occupation numbers (as that discussed above for the hypervalent sulfur) may be considered significant from the physico-chemical point of view.

\section{Interrelations between Hilbert-space and 3D analyses}

The Hilbert-space and the 3D-type analyses are much different conceptually, and exhibit quite different behaviour when the basis set is changed. The results of the Hilbert-space analysis is subjected to a strong basis-dependence, due to which one should compare only results obtained with the use of strictly the same basis set. (The large sensitivity of the Mulliken-populations to the basis applied is the best known example of this problem.) Also, the Hilbertspace results related to the individual atoms (pairs of atoms etc.) are lacking any complete basis-set limit: in principle, one can use a complete basis set located anywhere in the space, so the concept of atomic basis functions loses its meaning as the basis set becomes complete... The well-known consequence of these limitations is also that no meaningful results can be expected from a Hilbert-space analysis if the basis set contains diffuse functions that lack any pronounced atomic character.

From other side, the 3D results usually exhibit quite smooth convergence to some limiting values as the basis sets improve; they are however, rather sensitive to the manner in which the (disjunct or fuzzy) atomic domains are defined, that is to the actual values of the individual weight functions $w_{A}(\vec{r})$ in different points of the space.

The possibility to develop the analysis by using a common formalism, as it has also been done above, as well as the existence of some rules of "mapping" between the two schemes of analysis [22] stressed their common features. Nonetheless, in light of the sharp difference between them, it has been somewhat surprising to get the recent results $[13,23]$ permitting to find a specific interrelation between the 3D analysis and a specific case of the Hilbert-space analysis. The point is that the analysis described in the section 2 gives in the 3D case, in general, as many effective atomic orbitals for each atom as is the number of the MO-s in the system. The first AO-s among them are the orbitals of the effective minimal basis, while all together span the same subspace as the "atomic parts" $\psi_{i}^{A}$ of the original (canonical) molecular orbitals $\psi_{i}$. Therefore, the effective AO-s of all the atoms together form a basis, in which the molecular wave function can be expanded. As each of the orbitals of that basis belongs to a well-defined atom in the system, one can perform a conventional Hilbert-space analysis by using this special basis set.

Now, it appears that for that special basis set the Hilbert-space analysis gives exactly the same results as the 3D one: Mulliken's net $\left(q_{A A}\right)$, overlap 
$\left(q_{A B}\right)$ and gross $\left(Q_{A}\right)$ populations are strictly equal to their $3 \mathrm{D}$ counterparts and the same holds for the bond orders $\left(B_{A B}\right)$ - and therefore for the valences, too:

$$
\begin{gathered}
q_{A A}^{3 D}=q_{A A}^{L C E A O} \quad, \quad q_{A B}^{3 D}=q_{A B}^{L C E A O} \\
Q_{A}^{3 D}=Q_{A}^{L C E A O} \\
B_{A B}^{3 D}=B_{A B}^{L C E A O}
\end{gathered}
$$

where the abbreviation LCEAO means "linear combination of effective atomic orbitals". The proof of equalities (23) is simple but little involved, so here we refer only to the original publications [13,23].

This result indicates that the known problems with the Mulliken-type analyses are not related with the formalism, but rather to the basis set used: a basis which is good for energetic calculations is often not adequate for doing qualitative LCAO analyses, because contains a number of practically empty orbitals having significant overlaps with the occupied ones. ${ }^{6}$

\section{Conclusions and perspectives}

We have discussed that one can obtain effective atomic orbitals (generalized natural atomic hybrids) in quite different frameworks-Hilbert-space analysis, Bader-type or "fuzzy atoms" 3D analysis of the results of conventional quantum chemical calculations, as well as "fuzzy atoms" 3D analysis of those of the plane wave calculations. In all cases one can clearly distinguish between the orbitals of an effective minimal basis set and orbitals which are only insignificantly occupied. Although these orbitals do not strictly coincide with the free atomic ones, this observation makes a solid theoretical basis beyond our qualitative picture of molecular electronic structure, described in terms of minimal basis atomic orbitals having decisive participation in bonding, and may be considered as a quantum chemical manifestation of the octet rule. For strongly positive atoms like the hypervalent sulfur, some weakly occupied orbitals reflecting "back donation" can also be identified. (For sulfur they are of $d$-type, as could expected.) From the conceptual point of view, it is very important that atomic orbitals of characteristic shape can be

\footnotetext{
${ }^{6}$ Moreover, one may state that the fact that the $3 \mathrm{D}$ populations coincide with the Mulliken populations calculated in the basis of the effective AO-s, supports once again our point of view first expressed more than three decades ago [20], according to which the "halving" of the overlap populations characteristic for the Mulliken analysis is not an arbitrary choice and, therefore, Mulliken-populations have privileged mathematical importance in the framework of the LCAO formalism.
} 
obtained even by processing the results of plane wave calculations in which no atom-centered basis orbitals were applied. ${ }^{7}$

The different types of analyses (Hilbert-space and 3D) can be done on equal footing, performing quite analogous procedures, and they exhibit an unexpected interrelation: the Hilbert-space analysis performed in the basis of the effective atomic orbitals obtained in the 3D analysis gives results coinciding with those obtained directly in the 3D formalism.

Until now our attention has mainly been focused on obtaining the set of effective atomic orbitals in such a manner that the orbitals forming the effective minimal basis is distinguished from the other ones as sharply (as far as occupation numbers are concerned) as only possible. It has been observed that the minimal basis orbitals are resembling pure $s$ and $p$ ones for symmetric molecules and often represent some hybrids in the general case. It would be worth to study the detailed spatial form and directionality of the different effective minimal basis orbitals in order to be able to discuss the different steric effects, and perhaps relations to the VSEPR model too. ${ }^{8}$

Another perspective approach may be the investigation of the energetic effects which are connected with the distinction between the the full set of effective atomic orbitals and the effective minimal basis. One may, for instance, study how large energetic change takes place if one omits all - or some - of the weakly occupied orbitals from the basis of effective AOs. Alternatively, it may be of interest to calculate the (energetically) best minimal basis by direct variational calculation, instead of calculating the effective minimal basis orbitals from an a posteriori analysis of the results of an already accomplished calculation.

\section{Acknowledgments}

The author is indebted to his coauthors of different relevant papers, in particular to Dr. Pedro Salvador, Dr. Imre Bakó and Dr. András Stirling for the fruitful common work, and to the Hungarian Research Fund (OTKA) for the continuous financial support of his research during the last decades.

\footnotetext{
${ }^{7}$ As a Referee has stressed, the analyses of such type will be worth to be extended to systems like transition metals in typical bonding situations, and to check whether the results correspond to the appropriate generalizations of the octet rule - as "18 electron rule" - applied for such systems.

${ }^{8} \mathrm{~A}$ connection between hybridization and the VSEPR rules has already been discussed in $[24]$.
} 


\section{References}

1. G.G. Hall, Chairman's remarks, 5th International Congress on Quantum Chemistry, Montreal, 1985.

2. R. McWeeny, Rev. Mod. Phys. 32, 335 (1960)

3. R. Heinzmann, R. Ahlrichs, Theoret. Chim. Acta (Berl.) 42, 33 (1976)

4. K.R. Roby, Mol. Phys. 27, 81 (1974)

5. A.E. Reed, R. B. Weinstock and F. Weinhold, J. Chem. Phys. 83, 735 (1985)

6. A.E. Reed, L. A. Curtiss and F. Weinhold, Chem. Rev. 88, 899 (1988)

7. I. Mayer, Chem. Phys. Letters, 242, 499 (1995)

8. V. Magnasco, A. Perico, J. Chem. Phys. 47 (1967) 971.

9. I. Mayer, J. Phys. Chem. 100, 6249 (1996)

10. I. Mayer, Canadian J. Chem. 74, 939 (1996)

11. I. Mayer and P. Salvador, Chem. Phys. Letters, 383, 368 (2004)

12. I. Mayer, I. Bakó and A. Stirling, J. Phys. Chem. A, 115, 12733 (2011)

13. E. Ramos-Cordoba, P. Salvador and I. Mayer, J. Chem. Phys. 138, 214107 (2013)

14. R. Ponec, J. Math. Chem. 21, 323 (1997)

15. R. Ponec, D. L. Cooper and A. Savin, Chem. Eur. J. 14, 3338 (2008)

16. I. Mayer, Faraday Discuss. 135, 128 (2007)

17. R. Ponec, Faraday Discuss. 135, 129 (2007)

18. See e.g., pp 62-64 in I. Mayer, "Simple Theorems, Proofs, and Derivations in Quantum Chemistry", Kluwer Academic/Plenum Publishers, New York 2003.

19. I. Mayer, Program "EFFAO". May be downloaded from the web-site http://occam.chemres.hu/programs 
20. I. Mayer, Chem. Phys. Letters, 97, 270 (1983)

21. I. Mayer, J. Comput. Chem. 28, 204 (2007)

22. J.G. Ángyán, M. Loos and I. Mayer, J. Phys. Chem. 98, 5244-5248 (1994)

23. I. Mayer, Chem. Phys. Letters, 585, 198 (2013)

24. I. Mayer, Structural Chemistry 8, 309 (1997) 
Table 1

Partial valences of the different atoms in the $\mathrm{CH}_{3} \mathrm{SO}_{2} \mathrm{Cl}$ molecule calculated by using cc-pVTZ basis set.

\begin{tabular}{cccccc}
\hline \multirow{2}{*}{ Atom } & \multicolumn{5}{c}{ Valence contribution } \\
\cline { 2 - 6 } & $s$ & $p$ & $d$ & $f$ & Total \\
\hline $\mathrm{C}$ & 0.887 & 2.888 & 0.068 & 0.004 & 3.847 \\
$\mathrm{H}$ & 0.932 & 0.052 & 0.004 & - & 0.987 \\
$\mathrm{H}$ & 0.936 & 0.055 & 0.004 & - & 0.996 \\
$\mathrm{H}$ & 0.932 & 0.052 & 0.004 & - & 0.987 \\
$\mathrm{~S}$ & 0.880 & 2.961 & 1.777 & 0.199 & 5.816 \\
$\mathrm{O}$ & 0.264 & 1.776 & 0.029 & 0.002 & 2.070 \\
$\mathrm{O}$ & 0.264 & 1.776 & 0.029 & 0.002 & 2.070 \\
$\mathrm{Cl}$ & 0.040 & 0.961 & 0.046 & 0.004 & 1.051 \\
\hline
\end{tabular}




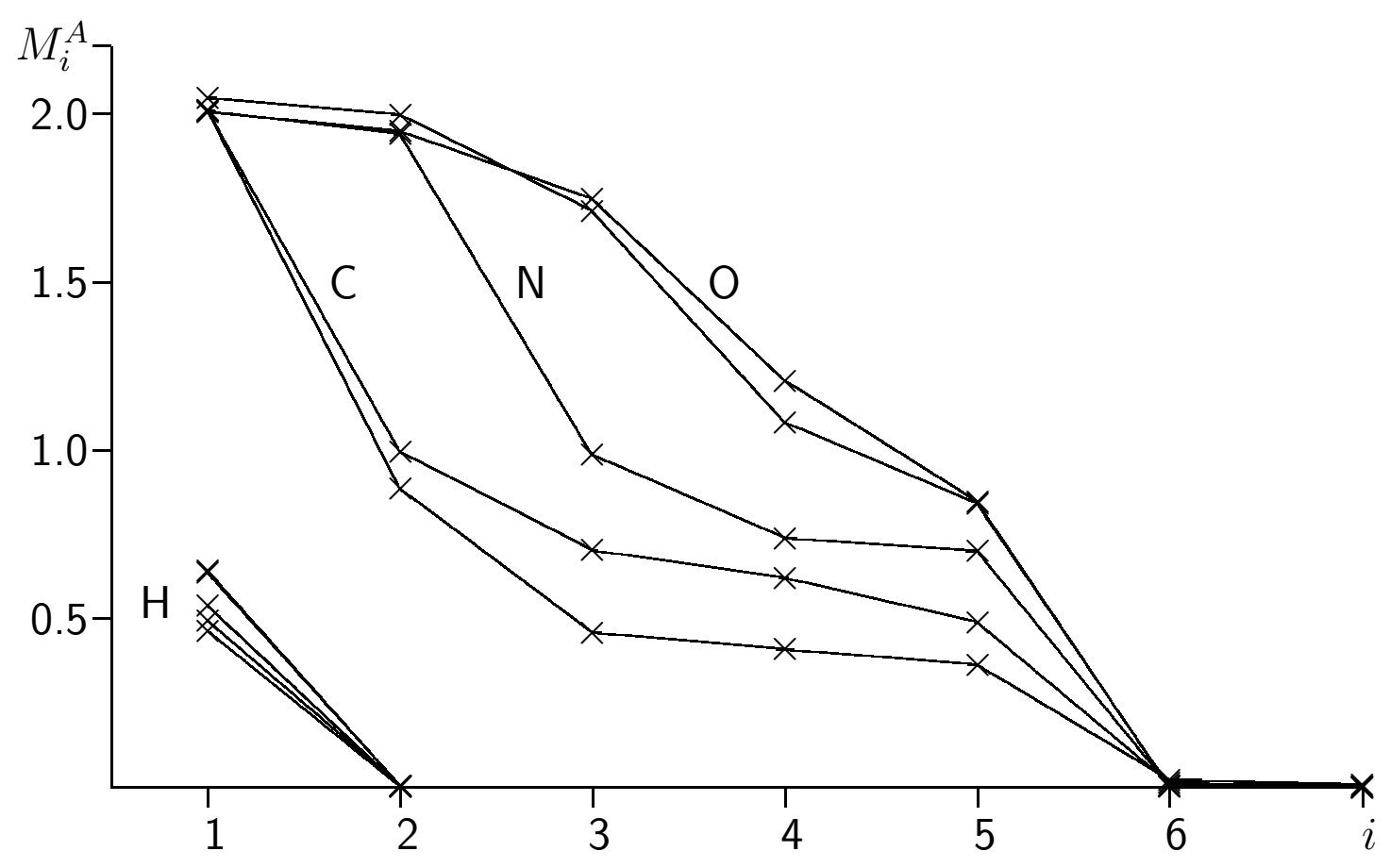

Figure 1

Occupation numbers of the effective AO-s in glycine molecule (cc-pVTZ) 


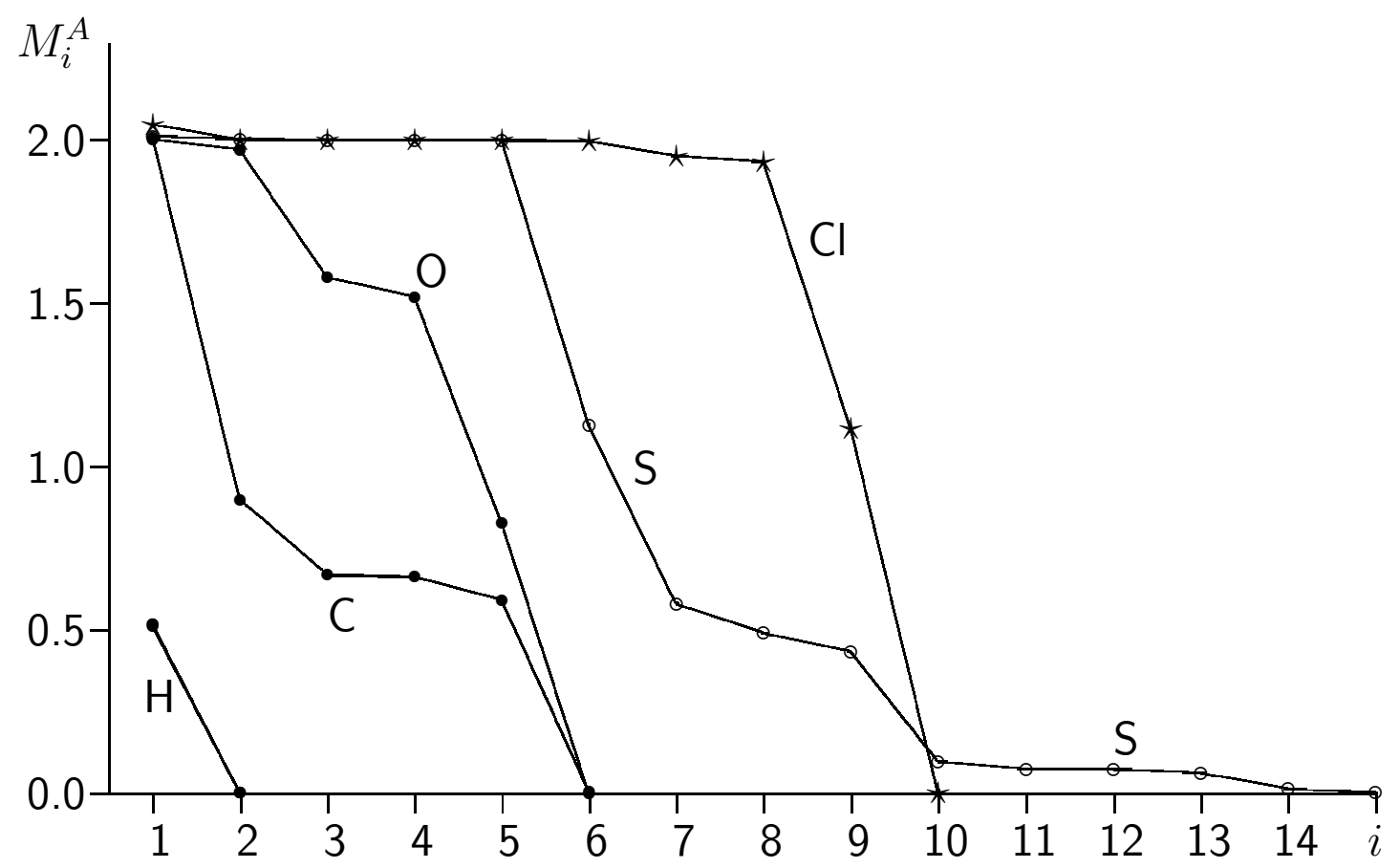

Figure 2

Occupation numbers of the effective $\mathrm{AO}-\mathrm{s}$ in the $\mathrm{CH}_{3} \mathrm{SO}_{2} \mathrm{Cl}$ molecule (cc-pVTZ) 

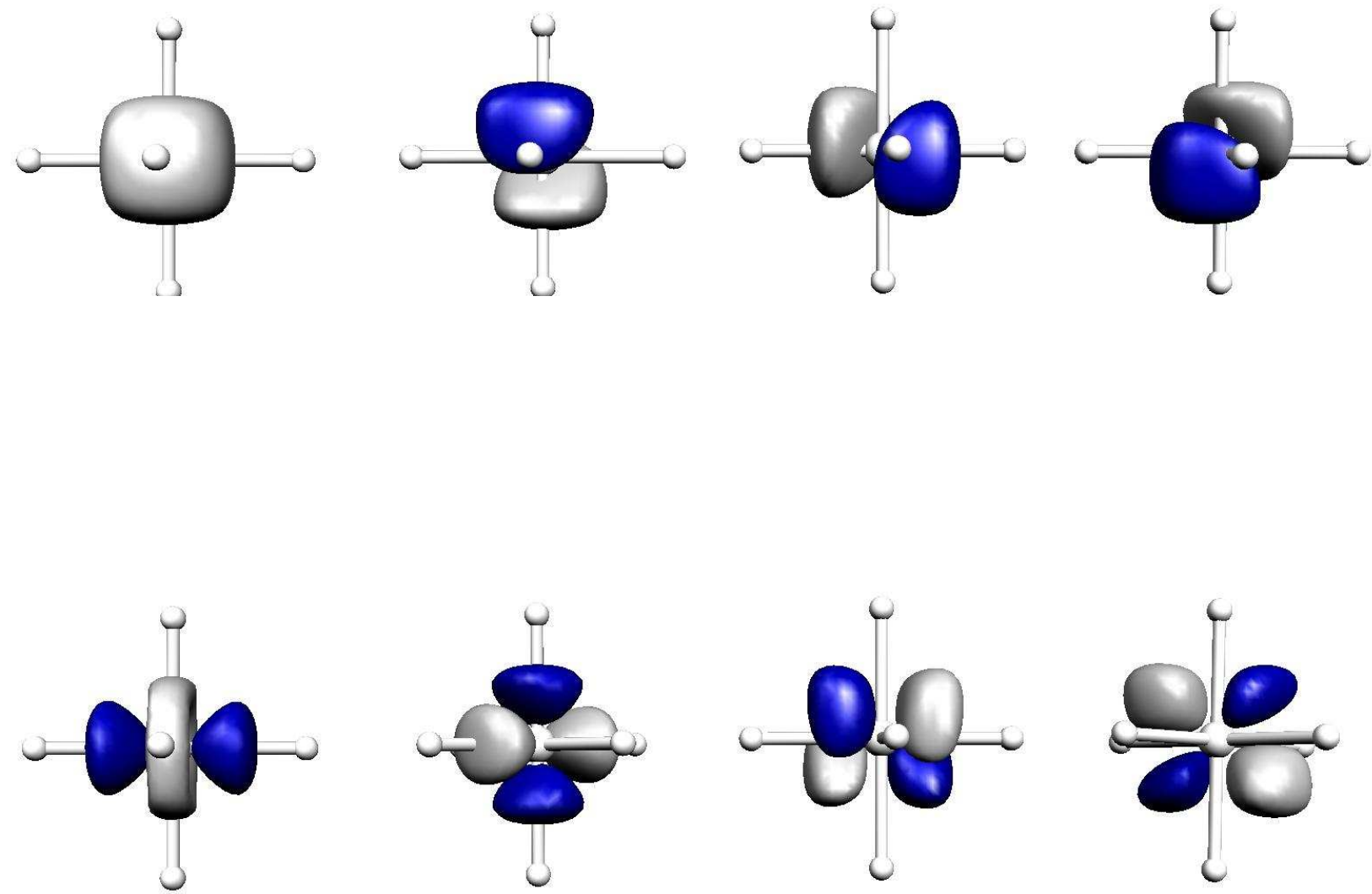

The strongly occupied valence and weakly occupied $d$-orbitals of the sulfur atom in the $\mathrm{SF}_{6}$ molecule, extracted from plane wave results. (Reproduced from Ref. 12.) 


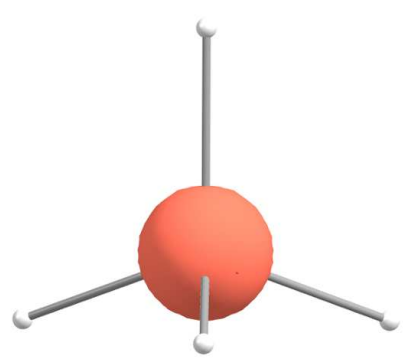

2.000

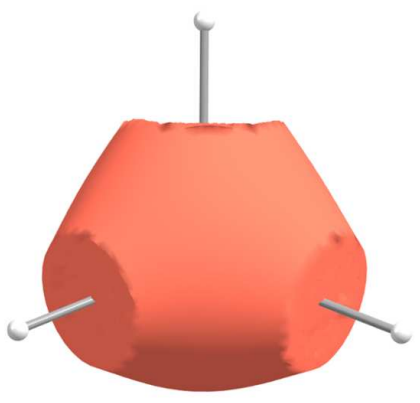

1.184

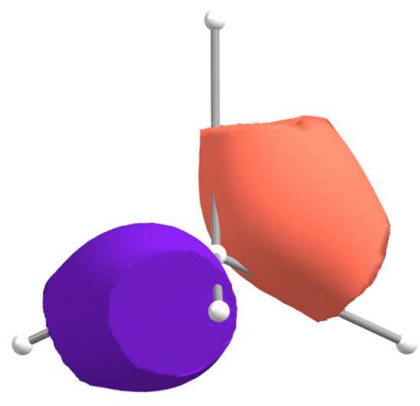

$3 \times 0.891$

\section{Figure 4}

Effective AO-s of the carbon atom in methane molecule and their occupation numbers: Bader-analysis. (Reproduced from Ref. 13.) 